\title{
Max-Plus Summation of Fenchel-Transformed Semigroups for Solution of Nonlinear Bellman Equations
}

\author{
William M. McEneaney * \\ Dept. of Math., Univ. of California, San Diego \\ La Jolla, CA 92093-0112, USA
}

April 22, 2004

\begin{abstract}
Max-plus methods have been explored for solution of first-order, nonlinear HamiltonJacobi-Bellman partial differential equations (HJB PDEs) and corresponding nonlinear control problems. These methods exploit the max-plus linearity of the associated semigroups. In particular, although the problems are nonlinear, the semigroups are linear in the max-plus sense. These methods have been used successfully to compute solutions. Although they provide certain advantages, they still generally suffer from the curse-of-dimensionality. The natural analog to the Laplace transform in ordinary spaces is the Fenchel transform over max-plus spaces, the range space being referred to as the dual space. One can transform the semigroup operators into operators on the dual space. There are natural operations on the transformed operators which may be used to construct solutions of nonlinear control problems. Natural building blocks correspond to transforms of operators for linear/quadratic problems. In this paper, a method for exploiting operations in the Fenchel transform space is used to develop a method for certain problems such that the computational growth in the most time-consuming portion of the computations can be hugely reduced. Although the curse-of-dimensionality is not entirely avoided, the computational cost reductions are very high for some classes of problems.
\end{abstract}

Key words: dynamic programming, partial differential equations, max-plus algebra, Legendre transform, Fenchel transform, semiconvexity, Hamilton-JacobiBellman equations, idempotent analysis.

*Dept. of Mechanical and Aerospace Eng. and Dept. of Math., University of California San Diego, wmceneaney@ucsd.edu Research partially supported by NSF grant DMS-0307229. 


\section{Introduction}

Hamilton-Jacobi-Bellman (HJB) partial differential equations (PDEs) arise naturally when one attempts to solve many nonlinear, continuous time/space control and estimation problems via dynamic programming. They specifically occur for problems where one is maximizing or minimizing over some input process (typically a controller or disturbance process). We look at control/estimation problems where the inputs have finite energy, and so the PDEs are first-order. These types of problems occur in optimal control and Robust $/ \mathrm{H}_{\infty}$ control and estimation. The HJB PDEs are nonlinear. For instance, in the $\mathrm{H}_{\infty}$ case, they typically contain a term which is quadratic in the gradient. Solution of such PDEs has presented a formidable challenge for many years. This challenge is most commonly summed up as the "curse-of-dimensionality".

In order to ground the effort, we will be concerned here with steady-state, fully nonlinear, first-order HJB PDEs. The solutions are typically nonsmooth, and so one must look for solutions in the weaker class of viscosity solutions. There is generally a unique viscosity solution, however for some problems one must use an additional criterion to select the correct viscosity solution [21], [23]. The computation of the solution of a nonlinear, steady-state, first-order PDE is typically quite difficult, and possibly even more so in the presence of the non-uniqueness mentioned above. Some previous works in the general area of numerical methods for these problems are [2], [6], [7], [11], [14], and the references therein. All these methods are, at the core, in the class of finite element methods.

In recent years, we have begun consideration of an entirely new class of numerical methods for HJB PDEs based on the linearity of the semi-group over the max-plus algebra [10], [17], [13], [18], [20], [19]. (Alternatively, one uses the min-plus algebra for minimizing control/estimation problems, but we will work only with the max-plus algebra and maximizing control/estimation problems so as not to be repetitious). This linearity had previously been noted in [16]. For purposes of completeness, we recall that the max-plus algebra is a commutative semi-field $(\mathbf{R} \cup\{-\infty\}, \oplus, \otimes)$ with the addition and multiplication operations given by

$$
a \oplus b=\max \{a, b\}, \quad a \otimes b=a+b
$$

where the operations are defined for $-\infty$ in the obvious way. Note that $-\infty$ is the additive identity, and 0 is the multiplicative identity. Note that it is not a field since the additive inverses are missing. (See [5], [1] among a burgeoning mass of literature related to the max-plus algebra.)

Another key ingredient in the development of this new class of max-plus-based numerical methods was the development of an appropriate max-plus function space (i.e. a space over the max-plus algebra) and corresponding max-plus basis for that space. Studies of abstract idempotent spaces (of which max-plus spaces form a subset) has begun in earnest in recent years (cf. [4], [15]). In fact this basis was first developed in [10] through the use of a semiconvex transform. The semiconvex transform is a slight modification of the 
Fenchel transform and convex duality relationship [10], [22]. (The Fenchel transform is an extension of the Legendre transform [3], [22].) In this paper, we will look more closely at this transform space, and the natural operations which may take place in it.

With max-plus-based methods for steady-state equations, one notes that the solutions of the HJB PDE are fixed points of the associated semigroup, that is $W=S_{\tau}[W]$ where $S_{\tau}$ is the semigroup with time-step $\tau$. Note that since 0 is the multiplicative identity, we can rewrite this as

$$
0 \otimes W=S_{\tau}[W] .
$$

In other words, $W$ is an eigenvector for $S_{\tau}$ corresponding to eigenvalue 0 . The solution is semiconvex ([18] among other references above). Letting $e$ be the semiconvex transform of $W$ (or, simply a truncation of the transform), one finds that $e$ satisfies

$$
e=\mathcal{B} \otimes e, \quad \text { or }, \quad 0 \otimes e=\mathcal{B} \otimes e
$$

where $\mathcal{B}$ is the transform of the operator $S_{\tau}$; when truncating the basis, $\mathcal{B}$ becomes a finite-dimensional matrix where $\otimes$ also represents max-plus matrix-vector multiplication.

In this paper, we will be concerned with natural operations on the transformed operator (specifically max-plus addition), and how these may be used as an aid in the solution of HJB PDEs. We note that in application of the max-plus-based numerical method given in the above references, there were two components to the computation: the computation of $\mathcal{B}$, and the computation of $e$ given $\mathcal{B}$. The computational time for the former greatly dominated that of the latter by an order of magnitude. Here, we will develop some techniques for operating on the transform operators themselves which will allow

us to construct a complex $\tilde{\mathcal{B}}$ from other, more easily computed, $\mathcal{B}$ 's. This can be used to develop a method for certain problems such that the computational cost in the most time-consuming portion of the computations can be hugely reduced. Interestingly, one has computational growth which is linear in a certain measure of problem complexity for which linear/quadratic problems have the minimal complexity. Although the curse-ofdimensionality is unavoidable, the computational cost reductions are very high for some classes of problems. A simple example in the class of problems where there is both an $L_{2}$ disturbance and a switching disturbance will be used for illustration. This will also motivate a very short discussion of a continuum viewpoint.

\section{Problem Set-up and Results Review}

The theory will be developed for steady-state nonlinear optimal control problems. In order to obtain explicit results, this problem class will be further reduced to the class of problems generally referred to a nonlinear $\mathrm{H}_{\infty}$ control problems. Of course, theory could be developed for other problem classes, but that would be well-beyond the scope of this paper. 
Consider a finite set of possible system dynamics indexed by $m \in \mathcal{M} \doteq\{1,2, \ldots, M\}$

$$
\dot{X}^{m}=f^{m}\left(X^{m}\right)+\sigma^{m}\left(X^{m}\right) w, \quad X_{0}^{m}=x \in \mathbf{R}^{n}
$$

where we note that all the systems have the same initial condition, and $w \in \mathcal{W}$ will be a (disturbance) input. To be specific, we take $\mathcal{W}=L_{2}^{\text {loc }}\left([0, \infty) ; \mathbf{R}^{k}\right)$ where the second argument indicates that the range of the disturbances lies in $\mathbf{R}^{k}$. A function, $w:[0, \infty) \rightarrow \mathbf{R}^{k}$ is in $L_{2}^{\text {loc }}$ if its restriction to any finite subinterval $[0, T]$ is in $L_{2}\left([0, T] ; \mathbf{R}^{k}\right)$. Specific assumptions on $f^{m}, \sigma^{m}$ will follow, but for now assume simply that the systems are sufficiently well-behaved such that there exist unique solutions for all $w \in \mathcal{W}$ and for all $x \in \mathbf{R}^{n}$. Denote a corresponding cost functional for each system by $J_{\tau}^{m}(x, w$.) where $\tau$ will denote the time horizon. Specifically, let

$$
J_{\tau}^{m}(x, w) \doteq \int_{0}^{\tau} h^{m}\left(X_{t}^{m}\right)-\frac{\gamma^{2}}{2}\left|w_{t}\right|^{2} d t
$$

where $X^{m}$ satisfies (4).

We will work with operators indexed by $\tau$ of the form

$$
S_{\tau}^{m}[\phi](x) \doteq \sup _{w \in \mathcal{W}}\left\{\int_{0}^{\tau} h^{m}\left(X_{t}^{m}\right)-\frac{\gamma^{2}}{2}\left|w_{t}\right|^{2} d t+\phi\left(X_{\tau}^{m}\right)\right\}(x)
$$

where $X^{m}$ satisfies (4). Note that $S_{\tau}^{m}$ is a semigroup in the standard algebra sense (i.e. $S_{\tau_{1}+\tau_{2}}^{m}[\phi]=S_{\tau_{1}}^{m}\left\{S_{\tau_{2}}^{m}[\phi]\right\}$ ). One typically finds that when the available storage (cf. [24], $[12]), W$, exists, it is a fixed point of the operator $S_{\tau}^{m}$ for any $\tau>0$. (See [12], [17], [18] among many others for proofs under standard assumptions.) In other words, the storage (a.k.a the value function)

$$
W^{m}(x) \doteq \sup _{0<\tau<\infty} \sup _{w \in L_{2}(0, \tau)} J_{\tau}^{m}(x, w)=\sup _{w \in \mathcal{W}} \sup _{0<\tau<\infty} J_{\tau}^{m}(x, w)
$$

is a fixed point of

$$
\phi=S_{\tau}^{m}[\phi] .
$$

In the $\mathrm{H}_{\infty} / L_{2}$-gain case under certain assumptions, it is known that the available storage is the unique fixed point in a class of continuous, nonnegative functions satisfying a certain quadratic growth condition, and we denote this class as $\mathcal{C}$ [21], [17], [18], [23]. For the moment, let us maintain a high level of generality, and not specify $\mathcal{C}$, but simply assume the existence of such a set (in which the available storage lies and is the unique fixed point); a specific example will be given below.

The available storage is also the unique viscosity solution in $\mathcal{C}$ of the corresponding HJB PDE. More specifically, in the case of (4),(5), one would have HJB PDE

$$
\begin{aligned}
0 & =H^{m}(x, \nabla \phi) \\
& =\sup _{w \in \mathbf{R}^{k}}\left[f^{m}(x) \cdot \nabla \phi+\left(\sigma^{m}(x) w\right)^{T} \nabla \phi+h^{m}(x)-\frac{\gamma^{2}}{2}|w|^{2}\right] \\
& =\sup _{w \in \mathbf{R}^{k}}\left[f^{m}(x) \cdot \nabla \phi+h^{m}(x)+\frac{1}{2 \gamma^{2}} \nabla \phi^{T} \sigma^{m}(x)\left(\sigma^{m}\right)^{T}(x) \nabla \phi\right] .
\end{aligned}
$$


with boundary condition $\phi(0)=0$ (i.e. $\phi$ being zero at the origin).

Thus, we see that one "typically" expects (for well-defined) integral functional problems, that the fixed point of the operator $S_{\tau}^{m}$ is identical to the viscosity solution of the HJB PDE (both being the available storage). We suppose for the remainder of the paper that there exists a unique solution of the corresponding fixed point problem $\phi=S_{\tau}^{m}[\phi]$ in class $\mathcal{C}$, and that this is also the unique viscosity solution of $0=H^{m}(x, \nabla \phi)$ in $\mathcal{C}$.

In order to indicate that the above results are not vacuous, a set of assumptions under which these results hold is now presented. We do not make these assumptions upfront for the entire paper, since the core concepts will be applicable to larger classes of problems. However, portions of the proofs will be specific to these assumptions. Since these assumptions only appear together, we will refer to this entire set of assumptions as Assumption Block $(H)$, and this is:

Assume that all $f^{m}$ and $\sigma^{m}$ are globally Lipschitz with the same constant $K<\infty$ (independent of $m), f^{m}(0)=0$, and that there exists $c \in(0, \infty)$ such that for any $m \in \mathcal{M}$

$$
(x-y)^{T}\left(f^{m}(x)-f^{m}(y)\right) \leq-c|x-y|^{2} \quad \forall x, y \in \mathbf{R}^{n} .
$$

Assume that there exists $c_{\sigma}<\infty$ such that for all $m \in \mathcal{M}$ and $x \in \mathbf{R}^{n}$, $\left|\sigma^{m}(x)\right| \leq c_{\sigma}$. Assume that there exist $C, \alpha<\infty$ such that for any $m \in \mathcal{M}$

$$
0 \leq h^{m}(x) \leq \alpha|x|^{2}, \quad\left|h^{m}(x)-h^{m}(y)\right| \leq C(1+|x|+|y|)|x-y| \quad \forall x, y \in \mathbf{R}^{n} .
$$

Lastly, assume that $\gamma^{2} / c_{\sigma}^{2}>\alpha / c^{2}$.

Theorem 2.1 Suppose Assumption Block $(H)$ holds. Then there exists a unique viscosity solution of (10), $\bar{W}^{m}$, in the class of continuous functions satisfying

$$
0 \leq \bar{W}^{m}(x) \leq c \frac{(\gamma-\delta)^{2}}{c_{\sigma}^{2}}|x|^{2}
$$

for sufficiently small $\delta>0$. Further, $\bar{W}^{m}=W^{m}$, the value (available storage) of control problem (4), (5), (7).

The above must now be placed in the context of semiconvex functions. Let $\mathcal{S}$ be the space of semiconvex functions mapping $\mathbf{R}^{n}$ into $\mathbf{R}^{-} \doteq \mathbf{R} \cup\{-\infty\}$ (cf. [10], [17], [13], [18], [19] among many more general references). As a reminder, recall that the space of semiconvex functions, $\mathcal{S}$, is defined as the set of $\psi: \mathbf{R}^{n} \rightarrow \mathbf{R}^{-}$such that for any $R<\infty$ there exists $C_{R}<\infty$ such that $\psi(x)+\frac{C_{R}}{2}|x|^{2}$ is convex over $\overline{B_{R}} \doteq \overline{B_{R}(0)}=\left\{x \in \mathbf{R}^{n}\right.$ : $|x| \leq R\}$. We refer to such a $C_{R}$ as a semiconvexity constant for $\psi$ over $\overline{B_{R}}$. Note that any $\psi \in \mathcal{S}$ is automatically locally Lipschitz [9]. For $R>0$, we denote the space of semiconvex functions for which the semiconvexity constant over $\overline{B_{R}}$ is $C$ as $\mathcal{S}_{R, C}$. Elements of $\mathcal{S}$ are 
Lipschitz over any ball, $\overline{B_{R}}$, and it will be useful to define the space $\mathcal{S}_{R, C, L}$ as the subspace of $\mathcal{S}_{R, C}$ consisting of functions which are Lipschitz with constant $L$ over $\overline{B_{R}}$.

One may view these spaces of semiconvex functions $\left(\mathcal{S}, \mathcal{S}_{R, C}, \mathcal{S}_{R, C, L}\right)$ as "vector spaces" over the max-plus commutative semi-field, and refer to them as max-plus vector spaces. In [1], [4], [15] a max-plus vector space is denoted as a moduloid. We now demonstrate a max-plus basis over $\mathcal{S}_{R, C, L}$. Note that as there is no concept of complete orthonormal set in a max-plus vector space. Instead we will use the term countable basis (or simply basis where countable is implied) of a max-plus space, $\mathcal{Y}$, to denote a countable set $\left\{\psi_{i}\right\}_{i=1}^{\infty} \subset \mathcal{Y}$ such that given any $y \in \mathcal{Y}$, there exists $\left\{a_{i}\right\}_{i=1}^{\infty} \subset \mathbf{R}^{-}$such that $y=\bigoplus_{i=1}^{\infty} a_{i} \otimes \psi_{i}$. For more on max-plus bases, see [10], [18].

Let $\phi \in \mathcal{S}_{R, C^{\prime}, L}$. Let $\left\{x_{i}\right\}$ be a countable, dense set over $\overline{B_{L / C^{\prime}}}$, and let symmetric $C-C^{\prime} I>0$ where (again) $C^{\prime}>0$ is a semiconvexity constant for $\phi$ over $\overline{B_{R}}$. Define

$$
\psi_{i}(x) \doteq-\frac{1}{2}\left(x-x_{i}\right)^{T} C\left(x-x_{i}\right)
$$

for each $i$. Then one finds (see [10] for details)

$$
\phi(x)=\bigoplus_{i=1}^{\infty}\left[a_{i} \otimes \psi_{i}(x)\right] \quad \forall x \in \overline{B_{R}}
$$

where each $a_{i} \doteq-\max _{x \in \overline{B_{R}}}\left[\psi_{i}(x)-\phi(x)\right]$. This is a countable max-plus basis expansion for $\phi$. More generally, the set $\left\{\psi_{i}\right\}$ forms a max-plus basis for the space $\mathcal{S}_{R, C^{\prime}, L}$. It is worth noting that scalar constants $C^{\prime}$ can be generalized to positive definite, symmetric matrices; see [18].

It is often the case that in the case of the integral functionals, the available storage (a.k.a. fixed-point of the semigroup, a.k.a. solution of the HJB PDE) is semiconvex. For example, one has the following [18].

Theorem 2.2 Consider the value/available storage for problem (4), (5), (7). Under Assumption Block $(H)$, this is semiconvex.

Suppose there are uniform semiconvexity and Lipschitz constants $C^{\prime}$ and $L$ for the $W^{m}$ over $\overline{B_{R}}$ for all $m \in \mathcal{M}$, i.e. the restrictions of the $W^{m}$ to $\overline{B_{R}}$ (also denoted as $W^{m}$ ) satisfy $W^{m} \in \mathcal{S}_{R, C^{\prime}, L}$ for all $m \in \mathcal{M}$. Let $C-C^{\prime} I>0$ and $\left\{x_{i}\right\}$ be dense over $\overline{B_{L / C^{\prime}}}$, and define the basis $\left\{\psi_{i}\right\}$ as above. Then

$$
W^{m}(x)=\bigoplus_{i=1}^{\infty}\left[a_{i}^{m} \otimes \psi_{i}(x)\right] \quad \forall x \in \overline{B_{R}}
$$

where

$$
a_{i}^{m} \doteq-\max _{x \in \overline{B_{R}}}\left[\psi_{i}(x)-W^{m}(x)\right]
$$


Throughout this paper, we will assume that such available storage functions actually have finite max-plus expansions, i.e. that

$$
W^{m}(x)=\bigoplus_{i=1}^{N}\left[a_{i}^{m} \otimes \psi_{i}(x)\right] \quad \forall x \in \overline{B_{R}}
$$

for some $N<\infty$. Although this assumption is completely unrealistic, it will greatly reduce the analysis - which seems acceptable in an introductory paper. Note that we used this same assumption in the early work on the use of max-plus linearity as a basis for a numerical method for solution of HJB equations (cf. [10], [17], [18]), and later proved convergence as the number of terms in basis expansion went to infinity [20], [19]. (Note that the full convergence analysis alone took an entire paper, and so it is neither possible nor useful to include such an analysis here.)

\section{Operating on the Transformed Operators}

In this paper, we will not be concerned with a numerical method for solution of HJB PDEs based on the max-plus eigenvector problem solution, but rather on the construction of matrices (the $\mathcal{B}$ matrices of Section 1) for max-plus eigenvector problems from other matrices whose max-plus eigenvector problems are analytically tractable, and the relationship of the constructed matrices to corresponding HJB PDEs. The following theorem makes a critical connection between the problems over the corresponding domains.

Theorem 3.1 Let $S_{\tau}^{m}$ be defined by (6) for each $m$ in some finite set $\mathcal{M}$. Suppose that for each $i \in\{1,2, \ldots, N\}$ and each $m \in \mathcal{M}$, there exists a finite basis expansion of $S_{\tau}^{m}\left[\psi_{i}\right]$, i.e. that

$$
S_{\tau}^{m}\left[\psi_{i}\right](x)=\bigoplus_{i=1}^{N} \mathcal{B}_{j, i}^{m} \otimes \psi_{j}(x) \quad \forall x \in \overline{B_{R}} .
$$

Define $\bar{S}_{\tau}[\phi]$ for any $\phi$ in the domain (to be specified for specific problems below) by

$$
\bar{S}_{\tau}[\phi](x)=\sup _{w \in \mathcal{W}}\left\{\max _{m \in \mathcal{M}}\left[\int_{0}^{\tau} h^{m}\left(X_{t}^{m}\right)-\frac{\gamma^{2}}{2}\left|w_{t}\right|^{2} d t+\phi\left(X_{\tau}^{m}\right)\right]\right\}
$$

for all $x \in \overline{B_{R}}$ where $X^{m}$ satisfies (4). Then

$$
\bar{S}_{\tau}\left[\psi_{i}\right](x)=\bigoplus_{j=1}^{N} \overline{\mathcal{B}}_{j, i} \otimes \psi_{j}(x)
$$

for all $x \in \overline{B_{R}}$ where

$$
\overline{\mathcal{B}}_{j, i}=\max _{m \in \mathcal{M}} \mathcal{B}_{j, i}^{m}=\bigoplus_{m \in \mathcal{M}} \mathcal{B}_{j, i}^{m}
$$

for all $i, j \in\{1,2, \ldots, N\}$. 
Proof. The proof is a simple manipulation given by

$$
\begin{aligned}
\bar{S}_{\tau}\left[\psi_{i}\right](x) & =\sup _{w \in \mathcal{W}}\left\{\max _{m \in \mathcal{M}}\left[l_{\tau}^{m}(x, w)+\psi_{i}\left(X_{\tau}^{m}\right)\right]\right\} \\
& =\max _{m \in \mathcal{M}} S_{\tau}^{m}\left[\psi_{i}\right](x)=\max _{m \in \mathcal{M}} \max _{j \in\{1,2, \ldots, N\}} \mathcal{B}_{j, i}^{m} \otimes \psi_{j}(x) \\
& =\max _{j \in\{1,2, \ldots, N\}}\left[\max _{m \in \mathcal{M}} \mathcal{B}_{j, i}^{m}\right] \otimes \psi_{j}(x)=\bigoplus_{j=1}^{N} \overline{\mathcal{B}}_{j, i} \otimes \psi_{j}(x)
\end{aligned}
$$

Remark 3.2 By simple modifications of [21] Theorems 2.1 and 2.6, one also has the following. Suppose Assumption Block $(H)$ holds. Then there is a unique continuous solution of $\bar{W}=\bar{S}_{\tau}[\bar{W}]$ satisfying

$$
0 \leq \bar{W}(x) \leq c \frac{(\gamma-\delta)^{2}}{c_{\sigma}^{2}}|x|^{2}
$$

for sufficiently small $\delta>0$.

Corollary 3.3 Suppose that the solution of $\bar{W}=\bar{S}_{\tau}[\bar{W}]$ lies in $\mathcal{S}_{R, C^{\prime}, L}$. (Recall from the discussion above that we are already assuming that the $W^{m}$ are in $\mathcal{S}_{R, C^{\prime}, L}$ and are fixed points of the $S_{\tau}^{m}$.) Further, assume that the expansion for $\bar{W}$ is also finite with $N$ coefficients which we denote as $\bar{W}=\bigoplus_{j=1}^{N} \bar{e}_{j} \otimes \psi_{j}$. Also assume that each $\psi_{j}$ is active in the sense that $\bigoplus_{i \neq j} \bar{e}_{j} \otimes \psi_{j} \neq \bigoplus_{j=1}^{N} \bar{e}_{j} \otimes \psi_{j}$ for any $i \leq N$. Then the vector of coefficients, $\bar{e}$ is the solution of the max-plus eigenvector equation $\bar{e}=\overline{\mathcal{B}} \otimes \bar{e}$ where $\overline{\mathcal{B}}_{j, i}=\bigoplus_{m \in \mathcal{M}} \mathcal{B}_{j, i}^{m}$ for all $j, i$.

ProOF. By assumption, for all $x \in \overline{B_{R}}$

$$
\bigoplus_{j=1}^{N} \bar{e}_{j} \otimes \psi_{j}(x)=\bar{W}(x)=\bar{S}_{\tau}[\bar{W}](x)=\bar{S}_{\tau}\left[\bigoplus_{i=1}^{N} \bar{e}_{i} \otimes \psi_{i}\right](x)=\bigoplus_{i=1}^{N} \bar{e}_{i} \otimes \bar{S}_{\tau}\left[\psi_{i}\right](x)
$$

which by Theroem 3.1

$$
=\bigoplus_{i=1}^{N} \bar{e}_{i} \otimes\left[\bigoplus_{j=1}^{N} \overline{\mathcal{B}}_{j, i} \otimes \psi_{j}(x)\right]=\bigoplus_{j=1}^{N}\left[\bigoplus_{i=1}^{N} \overline{\mathcal{B}}_{j, i} \otimes \bar{e}_{i}\right] \otimes \psi_{j}(x)
$$

Using the assumption that all the $\psi_{j}$ are active, this implies that $\bar{e}_{j}=\bigoplus_{i=1}^{N} \overline{\mathcal{B}}_{j, i} \otimes \bar{e}_{i} \forall j$, or equivalently, $\bar{e}=\overline{\mathcal{B}} \otimes \bar{e}$.

\section{The HJB PDE Limit Problems}

Now suppose that instead of desiring to solve for fixed points of the semigroups, one desires to solve related HJB PDEs. The assumptions in the theorems and corollary of 
Section 2 are all assumed to hold throughout this section. Let us consider here the HJB PDE

$$
\begin{aligned}
& 0=\widetilde{H}(x, \nabla \phi) \doteq \max _{m \in \mathcal{M}} H^{m}(x, \nabla \phi) \\
& \max _{m \in \mathcal{M}} \sup _{w \in \mathbf{R}^{k}}\left[f^{m}(x) \cdot \nabla \phi+\left(\sigma^{m}(x) w\right)^{T} \nabla \phi+h^{m}(x)-\frac{\gamma^{2}}{2}|w|^{2}\right] .
\end{aligned}
$$

Consider the sets of measurable processes with values in $\mathcal{M}$ given by

$$
\mathcal{M}^{p}=\{\mu:[0, \infty) \rightarrow \mathcal{M} \mid \text { measurable }\}, \quad \mathcal{M}_{T}^{p}=\{\mu:[0, T) \rightarrow \mathcal{M} \mid \text { measurable }\}
$$

Then by standard dynamic programming results under typical assumptions (cf. [21], [17], [20], [18]), one obtains the following theorem. A specific example of a class of dynamics, cost and set $\mathcal{C}$ is given in the remark just below the theorem statement.

Theorem 4.1 There exists a unique solution in some class $\mathcal{C}$ of PDE (19), and this viscosity solution is also the unique solution in $\mathcal{C}$ of

$$
\widetilde{W}=\widetilde{S}_{\tau}[\widetilde{W}]
$$

where

$$
\begin{aligned}
& \widetilde{S}_{\tau}[\phi](x) \doteq \sup _{\mu \in \mathcal{M}_{T}^{p}} \sup _{w \in \mathcal{W}_{\tau}}\left\{\int_{0}^{\tau} h^{\mu_{t}}\left(\widetilde{X}_{t}\right)-\frac{\gamma^{2}}{2}\left|w_{t}\right|^{2} d t+\phi\left(\widetilde{X}_{\tau}\right)\right\} \\
& \dot{\widetilde{X}}=\tilde{f}(\widetilde{X}, w, \mu) \doteq f^{\mu_{t}}\left(\widetilde{X}_{t}\right)+\sigma^{\mu_{t}}\left(w_{t}\right)
\end{aligned}
$$

This solution is also given by

$$
\widetilde{W}(x)=\sup _{\mu \in \mathcal{M}^{p}} \sup _{w \in \mathcal{W}} \sup _{T<\infty} \int_{0}^{T} h^{\mu_{t}}\left(\widetilde{X}_{t}\right)-\frac{\gamma^{2}}{2}\left|w_{t}\right|^{2} d t
$$

where $\widetilde{X}$ satisfies (23).

Remark 4.2 The general concepts underlying this work should hold for a variety of problems. Again however, in order to ground the analysis, specific assumptions under which Theorem 4.1 holds need to be specified. Theorem 4.1 holds under Assumption Block $(H)$ where $\mathcal{C}$ is the class of semiconvex functions satisfying $0 \leq \phi(x) \leq c \frac{(\gamma-\delta)^{2}}{c_{\sigma}^{2}}|x|^{2}$ for all $x$. See [18], [21] for details.

Note that the operators $\bar{S}_{\tau}$ do not necessarily form a semigroup, although they do form a sub-semigroup (i.e. $\bar{S}_{\tau_{1}+\tau_{2}}[\phi](x) \leq \bar{S}_{\tau_{1}} \bar{S}_{\tau_{2}}[\phi](x)$ for all $x$ and all $\phi$ in the domain). Further, it is easily seen that $S_{\tau}^{m} \leq \bar{S}_{\tau} \leq \widetilde{S}_{\tau}$ for all $\mathrm{m}$. 
With $\tau$ acting as a time-discretization step-size, let

$$
\begin{aligned}
\mathcal{M}^{p, \tau}= & \left\{\mu:[0, \infty) \rightarrow \mathcal{M} \mid \text { for each } n \in \mathbf{N} \cup\{0\}, \text { there exists } m_{n} \in \mathcal{M}\right. \\
& \text { such that } \left.\mu(t)=m_{n} \text { for } t \in[n \tau,(n+1) \tau)\right\},
\end{aligned}
$$

and for $T=\bar{n} \tau$ with $\bar{n} \in \mathbf{N}$ define $\mathcal{M}_{T}^{p, \tau}$ similarly but with domain $[0, T)$ rather than $[0, \infty)$. Let $\mathcal{M}^{N}$ denote the outer product of $\mathcal{M}, N$ times. Let $T=\bar{n} \tau$, and define

$$
\overline{\bar{S}}_{T}^{\tau}[\phi](x)=\max _{\left\{m_{k}\right\}_{k=0}^{\bar{n}-1} \in \mathcal{M}^{N}}\left\{\prod_{k=0}^{\bar{n}-1} S_{\tau}^{m_{k}}\right\}[\phi](x)
$$

where the $\prod$ notation indicates operator composition.

Roughly speaking the following theorem simply states that any nearly optimal (worst case) $w \in \mathcal{M}_{T}^{p}$ can be arbitrarily closely approximated (in terms of the cost) by a piecewise constant $w \in \mathcal{M}_{\tau}^{p, T}$ for some small $\tau$.

Theorem 4.3 Suppose that for any $x \in \mathbf{R}^{n}$, the origin lies in the interior of the convex hull of the set $\left\{f^{m}(x)\right\}_{m \in \mathcal{M}}$ and that Assumption Block $(H)$ holds. Given $T<\infty, R<\infty$ and $\varepsilon>0$, there exists $N \in \mathbf{N}$ sufficiently large such that letting $\tau=T / N$, one has $\widetilde{S}_{T}\left[W^{m}\right](x)-\varepsilon \leq \overline{\bar{S}}_{T}^{\tau}\left[W^{m}\right](x)$ for all $x \in \overline{B_{R}}$ and all $m \in \mathcal{M}$.

Sketch of Proof. There is insufficient space for the heuristically clear, but technically complex proof of Theorem 4.3; instead we present only a sketch of the main points. We note that the first assumption is essentially some sort of technical controllability assumption which is sufficient for the proof, but may not be necessary. The first step is to work with simple integrals. Consider some $g^{m} \in \mathbf{R}^{n}$ for all $m \in \mathcal{M}$, and suppose $0 \in\left\langle\left\{g^{m}\right\}_{m \in \mathcal{M}}\right\rangle^{\circ}$ (i.e. that the origin is in the interior of the convex hull of the set of the $\left.g^{m}\right)$. This guarantees that for any $\widehat{T}<\infty$ and $\Delta_{0} \in \mathbf{R}^{n}$, there exists $L<\infty$ and $\left\{\lambda_{m}^{0}\right\}_{m \in \mathcal{M}}$ such that $\lambda_{m}^{0} \in[0,1]$ for all $m, \sum_{m} \lambda_{m}^{0}=1$ and $\Delta_{0}=L \widehat{T} \sum_{m \in \mathcal{M}} \lambda_{m}^{0} g^{m}$. Then, given $\varepsilon>0, \widehat{T} \in(0, \infty)$ and $\Delta_{0},\left\{g^{m}\right\}, L$ as above and any $\mu \in \mathcal{M}_{T}^{p}$, there exist $N<\infty$, $\tau=\widehat{T} / N$ and $\bar{\mu} \in \mathcal{M}_{\widehat{T}}^{m, \tau}$ such that

$$
\left|\int_{0}^{\widehat{T}} g^{\bar{\mu}_{t}} d t-\left[\Delta_{0}+\int_{0}^{\widehat{T}} g^{\mu_{t}} d t\right]\right|<\varepsilon+\frac{L}{L+1}\left|\Delta_{0}\right| .
$$

In particular, one has $\int_{0}^{\widehat{T}} g^{\mu_{t}} d t=\widehat{T} \sum_{m \in \mathcal{M}} \lambda_{m}^{1} g^{m}$ for appropriate coefficients $\lambda_{m}^{1}$ (where $\lambda_{m}^{1} \in[0,1]$ for all $\left.m, \sum_{m} \lambda_{m}^{1}=1\right)$. The $g^{\bar{\mu}_{t}}$ process is created by setting the time-steps where $\bar{\mu}_{t}=m$ for each $m$ to approximate the fraction of time needed according to the $\left\{\lambda_{m}^{0}\right\}$ and $\left\{\lambda_{m}^{1}\right\}$ allocations, and this yields (25). One then approximates (23) over $[0, T)$ by holding the $\widetilde{X}$ terms on the right-hand side constant over each $[\widetilde{N} \widehat{T},(\widetilde{N}+1) \widehat{T})$ for $\widetilde{N} \in\{0,1, \ldots, \widehat{N}-1\}$ where $\widehat{N} \widehat{T}=T$ as

$$
\dot{\overrightarrow{\widetilde{X}}}_{t}=f^{\mu_{t}}\left(\widehat{\widetilde{X}}_{\widetilde{N} \widehat{T}}\right)+\sigma^{\mu_{t}}\left(\widehat{\widetilde{X}}_{\widetilde{N} \widehat{T}}\right) w_{t}
$$


For $\widehat{N}$ sufficiently large, $\max _{\widetilde{N}}\left|\widehat{\widetilde{X}}_{N \widehat{T}}-\widetilde{X}_{N \widehat{T}}\right|$ can be made arbitrarily small. (Since there is no a priori bound on $\|w\|$ in this formulation, an $L_{2}$ bound on near-optimal $w$ processes which holds under Assumption Block $(H)$ is used here.) A similar discretization approximation is employed with the state driven by the $\bar{\mu}$ process. Note that (26) takes the form $\dot{\overrightarrow{\widetilde{X}}}_{t}=g_{1}^{\mu_{t}}+g_{2}^{\mu_{t}} w_{t}$. The final discretization of $[0, T]$ is then with time-step $\tau=(T / \widehat{N}) / N$.

Now note that since $W^{m}, \bar{W} \in \mathcal{C}$, one has $\forall m$ (see Theorem 2.6, [21])

$$
\lim _{T \rightarrow \infty} \widetilde{S}_{T}\left[W^{m}\right]=\widetilde{W}, \quad \lim _{T \rightarrow \infty} \widetilde{S}_{T}[\bar{W}]=\widetilde{W}
$$

Also, for all $T<0$,

$$
\widetilde{W}=\widetilde{S}_{T}[\widetilde{W}]=\lim _{T \rightarrow \infty} \widetilde{S}_{T}[\widetilde{W}]
$$

uniformly on compact sets. By (27) and (28), given $R<\infty$ and $\varepsilon>0$, there exists $\widehat{T}<\infty$ such that for all $T \geq \widehat{T}$ and all $m \in \mathcal{M}$,

$$
\widetilde{S}_{T}[\widetilde{W}](x)-\varepsilon \leq \widetilde{S}_{T}\left[W^{m}\right](x) \quad \forall x \in \overline{B_{R}}
$$

Also note that

$$
\bar{W}=\bar{S}_{\tau}[\bar{W}]=\prod_{k=0}^{n-1} \bar{S}_{\tau}[\bar{W}] \geq \prod_{k=0}^{n-1} S_{\tau}^{m}[\bar{W}] .
$$

which using the fact that $\bar{W} \geq 0$ from Remark 3.2 and the monotonicity of $S_{\tau}^{m}[\cdot]$

$$
\geq \prod_{k=0}^{n-1} S_{\tau}^{m}[0]
$$

where 0 represents the function identically equal to zero. Since this is true for all $n$, one has (using Theorem 2.6, [21])

$$
\bar{W} \geq \lim _{n \rightarrow \infty} S_{n \tau}^{m}[0]=W^{m}
$$

for any $m \in \mathcal{M}$. On the other hand,

$$
\bar{W}=\prod_{k=0}^{n-1} \bar{S}_{\tau}[\bar{W}] \leq \prod_{k=0}^{n-1} \widetilde{S}_{\tau}[\bar{W}]=\widetilde{S}_{n \tau}[\bar{W}]
$$

which implies (using (27))

$$
\bar{W} \leq \lim _{T \rightarrow \infty} \widetilde{S}_{T}[\bar{W}]=\widetilde{W} .
$$

Combining (30) and (31), one has

$$
W^{m} \leq \bar{W} \leq \widetilde{W} \quad \forall m \in \mathcal{M} .
$$


Also, by definition it is obvious that

$$
\overline{\bar{S}}_{T}^{\tau}[\phi] \leq \widetilde{S}_{T}[\phi] \quad \forall \phi \in \mathcal{C} .
$$

Now, by Theorem 4.3 and (29), given $R<\infty$ and $\varepsilon>0$, there exist $T<\infty$ and $\bar{n}<\infty$ such that with $\tau=T / \bar{n}$, one has

$$
\widetilde{W}(x)-2 \varepsilon=\widetilde{S}_{T}[\widetilde{W}](x)-2 \varepsilon \leq \overline{\bar{S}}_{T}^{\tau}\left[W^{m}\right](x)
$$

which by (32), (33) and the monotonicity of $\widetilde{S}_{T}[\cdot]$

$$
\leq \overline{\bar{S}}_{T}^{\tau}[\bar{W}](x) \leq \widetilde{S}_{T}[\bar{W}](x) \leq \widetilde{S}_{T}[\widetilde{W}](x)=\widetilde{W}(x)
$$

$\forall x \in \overline{B_{R}}$. Since $\bar{W}(x)=\bar{S}_{\tau}[\bar{W}](x)=\left(\bar{S}_{\tau}\right)^{\bar{n}}[\bar{W}](x)=\overline{\bar{S}}_{T}^{\tau}[\bar{W}](x)$ on $\bar{B}_{R}$, this implies

Theorem 4.4 Given $R<\infty$ and $\varepsilon>0$, there exists $\tau>0$ such that

$$
\widetilde{W}(x)-2 \varepsilon \leq \bar{W}(x) \leq \widetilde{W}(x) \quad \forall x \in \overline{B_{R}}
$$

where $\widetilde{W}$ and $\bar{W}$ satisfy $\widetilde{W}=\widetilde{S}_{\tau}[\widetilde{W}]$ and $\bar{W}=\bar{S}_{\tau}[\bar{W}]$.

Recall from Corollary 3.3, that under the conditions given,

$$
\bar{W}(x)=\bigoplus_{j=1}^{N} \bar{e}_{j} \otimes \psi_{j}(x) \quad \forall x \in \overline{B_{R}}
$$

where the vector of coefficients, is the solution of the max-plus eigenvector equation $\bar{e}=\overline{\mathcal{B}} \otimes \bar{e}$ with $\overline{\mathcal{B}}=\bigoplus_{m \in \mathcal{M}} \mathcal{B}^{m}$. Thus Theorem 4.4 implies that one can solve HJB PDE (19) by solution of this eigenvector equation. If the $\mathcal{B}^{m}$ are such that they are easily computed (say by Riccati equations), then one has a method for computation of solutions of nonlinear HJB PDEs of the form (19) (or those which can be closely approximated by HJB PDEs of that form) where the most difficult portion of the computation, that of $\overline{\mathcal{B}}$, can be greatly simplified by representation of $\overline{\mathcal{B}}$ as a max-plus sum of the $\mathcal{B}^{m}$.

\section{A Simple Example}

As indicated above, a useful direction for application of this transform approach is as follows. One can solve simple linear/quadratic control problems through solution of the corresponding Riccati equations. Given a solution of the Riccati equation, one can construct the transformed operator (typically the discretized version thereof), $\mathcal{B}$, analytically with little effort. Thus, it is natural to consider HJB PDEs which can be represented or approximated by maxima of HJB PDEs corresponding to linear/quadratic problems. The simplest example of this approach will be discussed here to give the reader some flavor of a transformed operator construction approach. 
Consider the HJB PDE over $\mathbf{R}^{n}$ given by

$$
0=\max _{m \in \mathcal{M}}\left[\left(A^{m} x\right)^{T} \nabla W\right]+\frac{1}{2} x^{T} D x+\frac{1}{2} \nabla W^{T} \Sigma \nabla W
$$

where $\Sigma \doteq \sigma \Gamma^{-1} \sigma^{T}$, and we assume $D, \Gamma$ symmetric, positive definite, and Assumption Block $(H)$ with $f^{m}(x)=a^{m} x, \sigma^{m}(x)=\sigma, h^{m}(x)=\frac{1}{2} x^{T} D x$ for all $m$, and $\frac{\gamma^{2}}{2}|w|^{2}$ replaced by the more general for $\frac{1}{2} w^{T} \Gamma w$. (Also, as above, we assume that $\mathcal{M}$ is a finite set.) The goal here is to demonstrate the mechanics of a procedure for solution of (35). HJB PDE (35) corresponds to a control problem which has both an unknown $L_{2}$ disturbance process and a switching disturbance process given by

$$
\begin{aligned}
& \dot{X}_{t}=A^{\mu_{t}} X_{t}+\sigma w_{t}, \quad X_{0}=x \in \mathbf{R}^{n} \\
& W(x)=\sup _{\tau \in[0, \infty)} \sup _{w \in L_{2}} \sup _{\mu \in \mathcal{M}^{p}} \int_{0}^{\tau}\left[\frac{1}{2} X_{t}^{T} D X_{t}-\frac{1}{2} w_{t}^{T} \Gamma w_{t}\right] d t .
\end{aligned}
$$

For each $m \in \mathcal{M}$, the corresponding HJB PDE and semigroups are

$$
0=\left(A^{m} x\right)^{T} \nabla W+\frac{1}{2} x^{T} D x+\frac{1}{2} \nabla W^{T} \Sigma \nabla W
$$

and

$$
\begin{aligned}
& S_{\tau}^{m}\left[\psi_{i}\right](x)=\sup _{w \in L_{2}}\left\{\int_{0}^{\tau}\left[\frac{1}{2} X_{t}^{m T} D X_{t}^{m}-\frac{1}{2} w_{t}^{T} \Gamma w_{t}\right] d t+\psi_{i}\left(X_{\tau}^{m}\right)\right\} \\
& \dot{X}_{t}^{m}=A^{m} X_{t}^{m}+\sigma w_{t}, \quad X_{0}=x .
\end{aligned}
$$

The solutions will be denoted by $V_{i}^{m}(0, x)=S_{\tau}^{m}\left[\psi_{i}\right](x)$ where the $V_{i}^{m}:[0, \tau] \times \mathbf{R}^{n}$. Letting $\psi_{i}(x)=-\frac{1}{2}\left(x-x_{i}\right)^{T} C\left(x-x_{i}\right)$, one may assume (w.l.o.g.) that the $V_{i}^{m}$ take the form $V_{i}^{m}(t, x)=\frac{1}{2}\left(x-\Lambda_{t}^{m} x_{i}\right)^{T} Q_{t}^{m}\left(x-\Lambda_{t}^{m} x_{i}\right)+\frac{1}{2} x_{i}^{T} R_{t}^{m} x_{i}$. One then finds terminal conditions

$$
Q_{\tau}^{m}=C, \quad \Lambda_{\tau}^{m}=I, \quad R_{\tau}^{m}=0
$$

and ordinary differential equations (ODEs)

$$
\begin{aligned}
& \dot{Q}^{m}=\left[D+Q^{m} \Sigma Q^{m}-\left(\left(A^{m}\right)^{T} Q^{m}+Q^{m} A^{m}\right)\right] \\
& \dot{\Lambda}^{m}=\left(A^{m}-Q^{m-1} D\right) \Lambda, \quad \dot{R}^{m}=-\Lambda^{m T} D \Lambda^{m} .
\end{aligned}
$$

It is important to note that none of these ODEs depend on $x_{i}$. They only need to be solved once for each $m \in \mathcal{M}$. Noting that $S_{\tau}^{m}\left[\psi_{i}\right](x)=V_{i}^{m}(0, x)$, and that one then has $\mathcal{B}_{i, j}^{m}=-\max _{x \in B_{R}}\left\{\psi_{i}(x)-V_{j}^{m}(0, x)\right\}$, one can show that

$$
\mathcal{B}_{i, j}^{m}=q_{i}^{1}+q_{j}^{2}+\gamma_{i}^{T} l_{j}^{1}-\alpha_{i}^{T} l_{j}^{2}
$$

where we drop the $m$ superscripts for notational simplicity)

$$
\begin{array}{ll}
q_{i}^{1}=\frac{1}{2}\left(\alpha_{i}^{T} C \alpha_{i}-\gamma_{i}^{T} Q_{0} \gamma_{i}^{T}\right), & q_{i}^{1}=\frac{1}{2}\left(\beta_{j}^{T} C \beta_{j}-\delta_{j}^{T} Q_{0} \delta_{j}^{T}\right)+\frac{1}{2} x_{j}^{T} R_{0} x_{j} \\
l_{j}^{1}=q_{0} \delta_{j}, \quad l_{i}^{2}=C \beta_{i} & \\
\alpha_{i}=\left[\left(C-Q_{0}\right)^{-1} C-I\right] x_{i}, & \gamma_{i}=\left(C-Q_{0}\right)^{-1} C x_{i} \\
\beta_{j}=\left(C-Q_{0}\right)^{-1} Q_{0} \Lambda_{0} x_{j}, & \delta_{j}=\left[\left(C-Q_{0}\right)^{-1} Q_{0}+I\right] x_{j} .
\end{array}
$$


Note that the only computation that needs to be done for all pairs $(i, j)$ is $(40)$. This implies that only $4 n+1$ floating point operations need be performed for each of the $N^{2}$ pairs $(i, j)$. The other operations above are performed only once for each of the $N$ single indices $i$. (In practice, it has been observed that one does not need to compute $\mathcal{B}_{i, j}^{m}$ for all pairs $(i, j)$. The solution obtained by computing only those $\mathcal{B}_{i, j}^{m}$ such that $x_{i}-x_{j}$ is relatively small is identical to the solution obtained by computing the entire matrix. That the solution is identically the same rather than merely "close" is a typical property in idempotent algebras.) One then obtains

$$
\overline{\mathcal{B}}_{i, j}=\max _{m \in \mathcal{M}} \mathcal{B}_{i, j}^{m} \quad \forall i, j
$$

Lastly, one solves $\bar{e}=\overline{\mathcal{B}} \otimes \bar{e}$. This max-plus eigenvector problem may be solved via the power method (see, for instance, [18], [19], [20], [5]). This converges exactly in a finite number of steps to the unique eigenvector $\bar{e}$.

A simple example with $\mathcal{M}=\{1,2\}$ has been included. The computation takes about 5 seconds on a standard desktop PC. In the example,

$$
A^{1}=\left[\begin{array}{ll}
-1 & 0.5 \\
0.1 & -1
\end{array}\right], \quad A^{2}=\left[\begin{array}{cc}
-1 & 0.1 \\
0.5 & -1
\end{array}\right], \quad D=\left[\begin{array}{ll}
1.5 & 0.2 \\
0.2 & 1.5
\end{array}\right], \quad \Sigma=\left[\begin{array}{cc}
0.4 & -0.1 \\
-0.1 & 0.4
\end{array}\right]
$$

Perhaps we should note that the solution is not the piecewise combination of the solutions of the constituent LQ problems. The value function, its two partials and a backsubstitution error are plotted in figures 1 and 2. (The backsubstitution error is computed through approximation of the gradient via simple (perhaps overly simple) first-order differencing, and substitution into the HJB PDE.) The sharp cleft in the error plot is due to a discontinuity in the gradient of the value function. The rise at the corners opposite this cleft indicates that some additional basis functions should have been added to cover this region; thus the user can determine when one needs to extend this set.
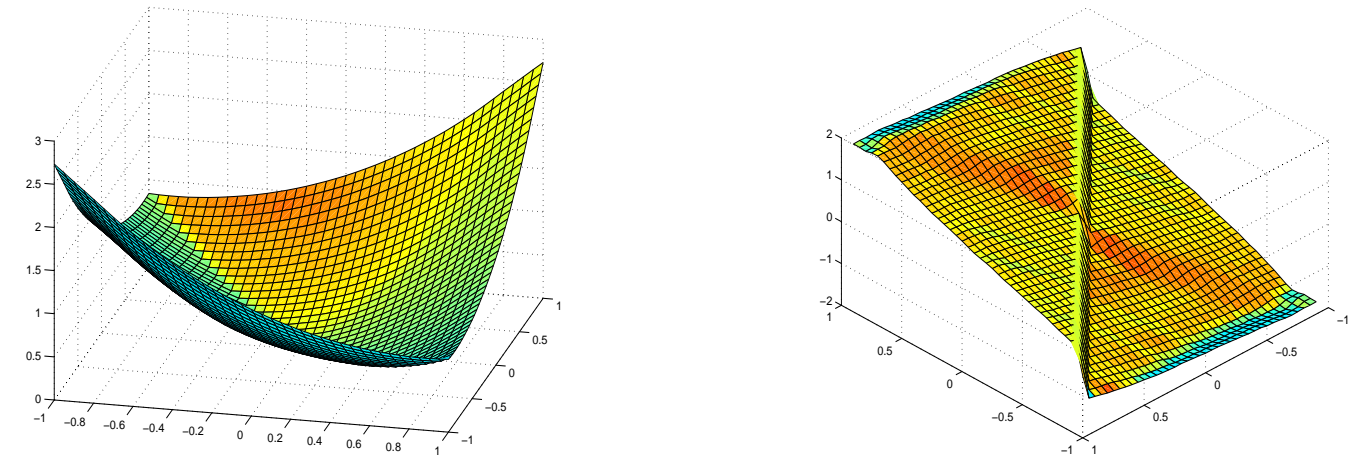

Figure 1: Value function and a partial 

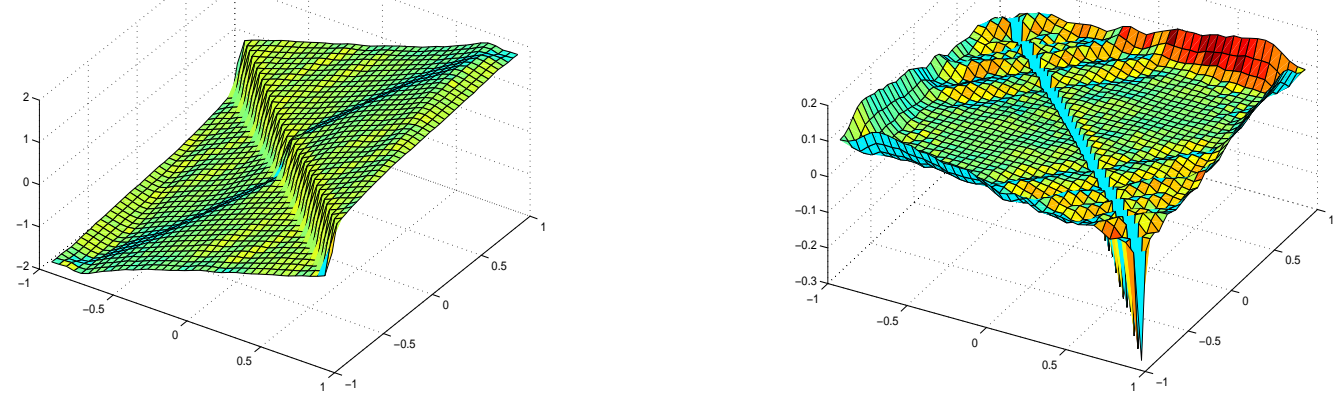

Figure 2: A partial and backsubstitution error

It is worth noting that when one uses HJB PDEs that are quadratic functions of $x, \nabla W$, the corresponding transformed operators, $\mathcal{B}^{m}$ are quadratic functions. In particular, for the very simple example HJB PDE above (with no linear or $0^{\text {th }}$ order terms), one finds that the $\mathcal{B}^{m}$ take the simple quadratic form

$$
\mathcal{B}_{i, j}^{m}=\frac{1}{2}\left(x_{i}^{T}, x_{j}^{T}\right) G^{m}\left(x_{i}^{T}, x_{j}^{T}\right)^{T}
$$

for a matrix $G^{m}$ which is easily computed.

\section{References}

[1] F.L. Baccelli, G. Cohen, G.J. Olsder and J.-P. Quadrat, Synchronization and Linearity, John Wiley (1992).

[2] M. Bardi and I. Capuzzo-Dolcetta, Optimal Control and Viscosity Solutions of Hamilton-Jacobi-Bellman Equations, Birkhauser, Boston, 1997.

[3] F.H. Clarke, Optimization and Nonsmooth Analysis, John-Wiley (1983).

[4] G. Cohen, S. Gaubert and J.-P. Quadrat, Duality of Idempotent Semimodules, Linear Algebra and Applications (to appear).

[5] R.A. Cuninghame-Green, Minimax Algebra, Lecture Notes in Economics and Mathematical Systems 166, Springer (1979).

[6] P. Dupuis and M. Boué, Markov chain approximations for deterministic control problems with affine dynamics and quadratic cost in the control, SIAM J. on Numerical Analysis, 36 (1999), pp. 667-695. 
[7] P. Dupuis and A. Szpiro, Convergence of the optimal feedback policies in a numerical method for a class of deterministic optimal control problems, SIAM J. Control and Optim. 40 (2001), pp. 393-420.

[8] W.H. Fleming, Max-Plus Stochastic Processes (2003), Applied Math. and Optim., (to appear).

[9] W.H. Fleming, Functions of Several Variables, Springer-Verlag (1977).

[10] W.H. Fleming and W.M. McEneaney, A max-plus based algorithm for an HJB equation of nonlinear filtering, SIAM J. Control and Optim., 38 (2000), 683-710.

[11] W.H. Fleming and H.M. Soner, Controlled Markov Process and Viscosity Solutions, Springer-Verlag, New York, 1993.

[12] J.W. Helton and M.R. James, Extending $\mathbf{H}_{\infty}$ Control to Nonlinear Systems, SIAM (1999).

[13] M.R. James and W.M. McEneaney, Max-plus approximation methods in partially observed $\mathrm{H}_{\infty}$ control, 38th IEEE Conf. on Decision and Control, 3011-3016.

[14] H.J. Kushner and P. Dupuis, Numerical methods for Stochastic Control Problems in Continuous Time, Springer Verlag, New York, 1992.

[15] G.L. Litvinov, V.P. Maslov and G.B. Shpiz, Idempotent Functional Analysis: An Algebraic Approach, Mathematical Notes, 69, No. 5 (2001), 696-729.

[16] V.P. Maslov, On a new principle of superposition for optimization problems, Russian Math. Surveys, 42 (1987) 43-54.

[17] W.M. McEneaney and M. Horton, Max-plus eigenvector representations for nonlinear $\mathrm{H}_{\infty}$ value functions, 37th IEEE Conf. on Decision and Control (1998), 3506-3511.

[18] W.M. McEneaney, Max-Plus Eigenvector Representations for Solution of Nonlinear $\mathrm{H}_{\infty}$ Problems: Basic Concepts, IEEE Trans. Auto. Control 48, (2003) 1150-1163.

[19] W.M. McEneaney, Max-Plus Eigenvector Representations for Solution of Nonlinear $\mathrm{H}_{\infty}$ Problems: Error Analysis, SIAM J. Control and Optim. (to appear).

[20] W.M. McEneaney Error Analysis of a Max-Plus Algorithm for a First-Order HJB equation, Stochastic Theory and Control, Proceedings of a Workshop held in Lawrence, Kansas, October 18-20, 2001, Lecture Notes in Control and Information Sciences, Springer-Verlag, B.Pasik-Duncan (Ed).

[21] W.M. McEneaney, A Uniqueness result for the Isaacs equation corresponding to nonlinear $\mathrm{H}_{\infty}$ control, Math. Controls, Signals and Systems, 11 (1998), 303-334.

[22] R.T. Rockafellar and R.J. Wets, Variational Analysis, Springer-Verlag (1997). 
[23] P. Soravia, $\mathrm{H}_{\infty}$ control of nonlinear systems: differential games and viscosity solutions, SIAM J. Control and Optim., 34 (1996), 1071-1097.

[24] A.J. van der Schaft, " $L_{2}-$ Gain analysis of nonlinear systems and nonlinear state feedback $\mathrm{H}_{\infty}$ control, IEEE Trans. Auto. Control, 37 (1992) 770-784. 